\title{
A Novel Framework for Ensuring Online Exam Authentication at Taibah University
}

\author{
Jawad H Alkhateeb \\ College of Computer Science and Engineerring, Taibah University, AlMadinah AlMunawarah, Kingdom of Saudi Arabia.
}

ABSTRACT - Using the internet technologies in teaching and learning is growing up rapidly. The main vital components in online learning are submitting the student's assessments and conducting the online exams. The main challenge in conducting the online exams is ensuring the authentication of the online exam takers. In this paper, a secure framework using biometrics for online exam takers at Taibah University is proposed. The secure framework uses two levels of authentication. First, the login level via the exam taker user name and his/her password. Second, the exam taker biometric authentication based on his/her iris recognition. In order to validate the proposed framework, various experiments were carried out. The proposed framework yields to an attractive result of $85.5 \%$ as recognition rate.
ARTICLE HISTORY

Received: 18 Feb 2020

Accepted: 07 April 2020

\section{KEYWORDS}

Online exam

biometrics

feature Extraction

DCT.

\section{INTRODUCTION}

Using and applying biometric authentication is a must nowadays for security purposes. The biometric authentication is known as the security manner for verifying the real person. This verification relies on the unique person biological characteristics. The biometric authentication has its own mechanism which is done by comparing biometric captured data to the stored data in any database. The use of biometric authentication is a process of managing the access to any resource from various recourses. These resources are categorized into two main classes: digital and physical resources. The resources are wide in real; they can be computer device, labs, cars, smart phones, car parking, room buildings, etc. There are different types of biometric authentications such as finger print or iris recognition. Conducting exams is necessary in different domains. These domains can be classified into schools, colleges, universities, and companies. The schools, colleges, and universities conduct their exams for education puposes. Also, both colleges, and universities conduct their exam for training certificatis. On the other hand, the companies conduct their exams for the purpose of recruitment. Futrthermore, there is anothore educatioal firm which conducts onlines exam. This firm is kown as homeschooling or home education (Ramu, T and Arivoli. 2013). Basically, the homescooling is the kids education at home which is usually conducted by a parent, teacher, or an online teacher. Homescooling processes is popular the United states, United Kingdom, Australia, New Zealand, and other common wealth Commonwealth countries. The homeshooling students conduct their exams in a secure mechanism. This mechanism is known an homeschool testing service. The homeschooling testing services provide a place for all student to condcut their exams by using the onsite testing. Basiclly, the Onsite testing mechanism is a unique opportunity for student to share the school classroom situation experience testing. By using such an environment the student goes to any testing center location, where he/she meets with other group of students. There are a lot of families in KSA doing homeschholon for their kids. The students do their exams in a culture center which is suggested by the school.

There are two types of exams either paper based or online exams. By comparing both types of exams, it is concluded that the paper based exams are slower than the online exams in the manner of evaluating and grading exams. In order to speed up the evaluating process for exam takers, there is a huge need for online exams to replace the paper exams. This is can be done by using the rapid growth of internet and its technologies. Basically, using biometric authentication is to manage the access as mentioned earlier and to prevent unauthorized people from accessing. The unauthorized people are known as fraud identity. Mainly, the fraud identity is classified into three main classes:

1. Theft identity

2. Sharing identity

3. Denial identity

The theft identity occurs while the user identity is hijacked by a fraudster to be used in conducting any malicious activity. The sharing identity which is known as gift identity occurs when a user is willing to share his /her credentials with other users. Finally, the denial identity occurs while the unauthorized user conducts illegal actions (Alkhateeb Jawad 2018).

This paper proposes a new framework to the online exam takers in Taibah University. The exam takers at Taibah University can be regular student, or distance learning student. Both regular and distance learning student use the 
blackboard environment in conducting their online exams (Traore Isaa, et al. 2017). The process of conducting an online exam at Taibah University is summarized as follows:

1. The student exam taker login in into his/her blackboard account as illustrated in figure 1. Usually, the user name is the student university number
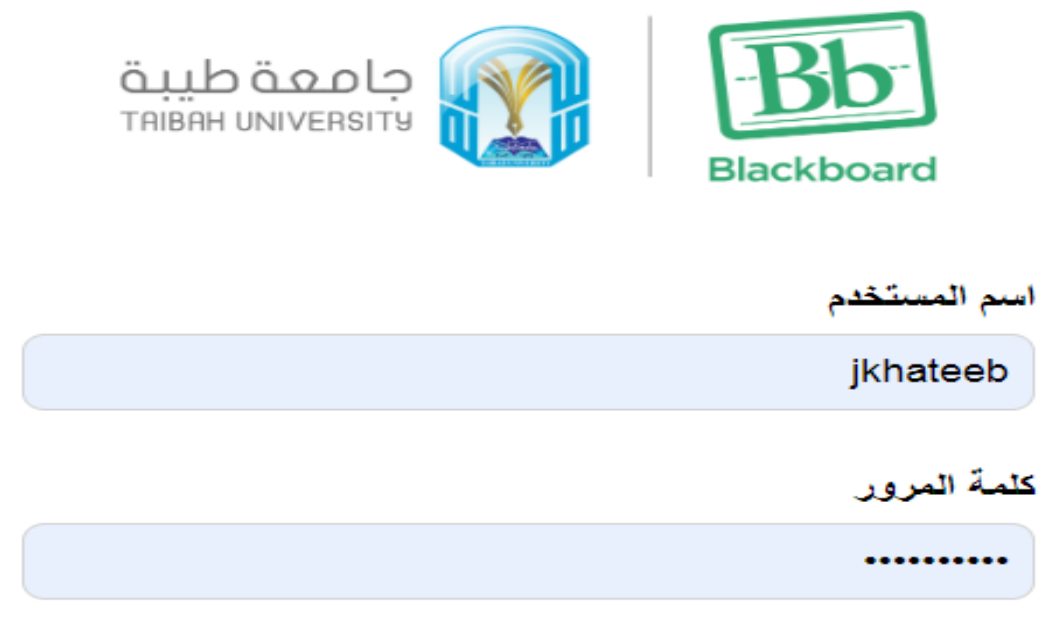

\section{تسجيل الهخمل}

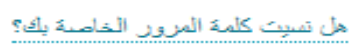

Figure 1. Blackboard log in process.

2. After a success login in to the blackboard, the exam taker goes to his/her course in order to choose the required exam by clicking on it as illustrated in figure 2 .

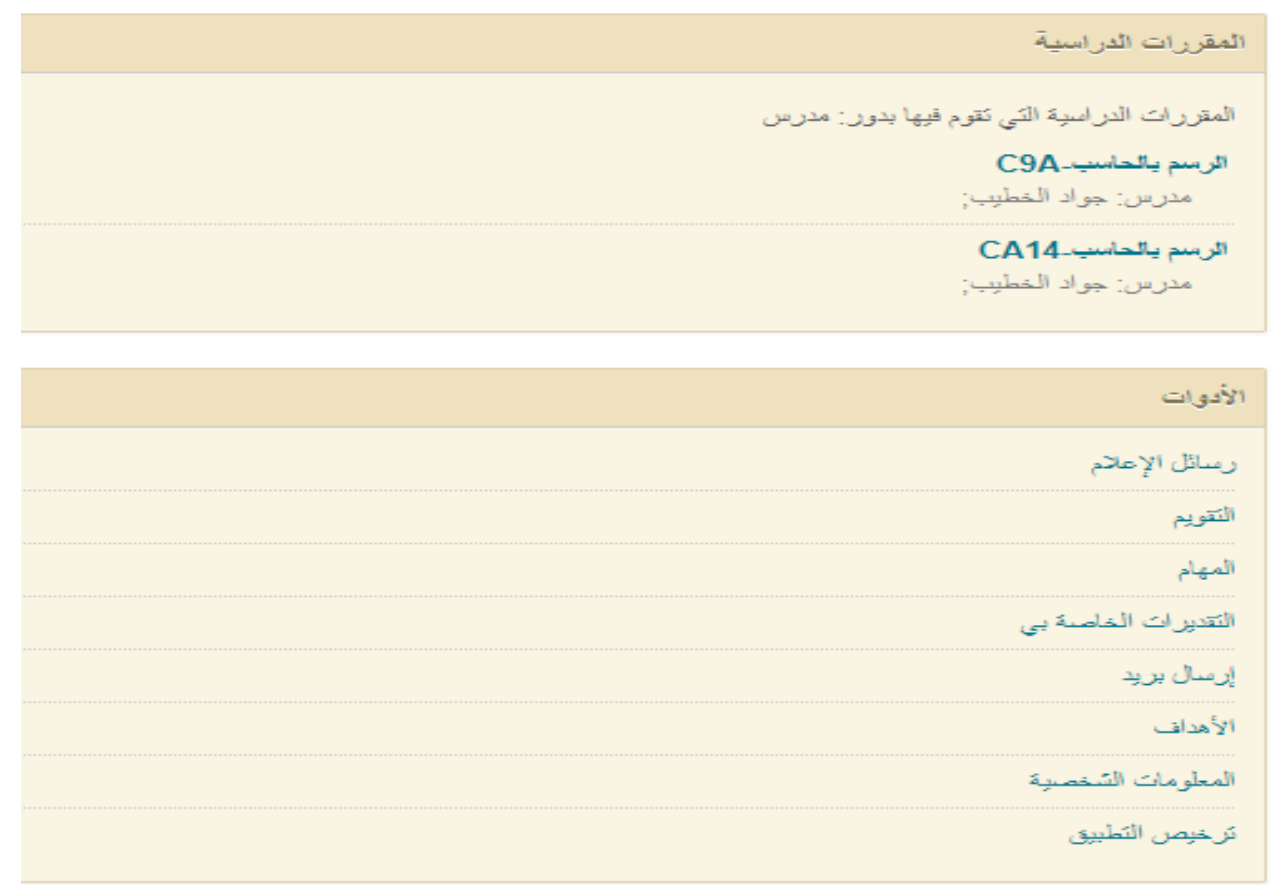

Figure 2. Choosing the required exam.

3. Finally, the exam taker needs to enter the password for the required exam. This password is assigned by the course instructor for security purposes as illustrated in figure 3 . 


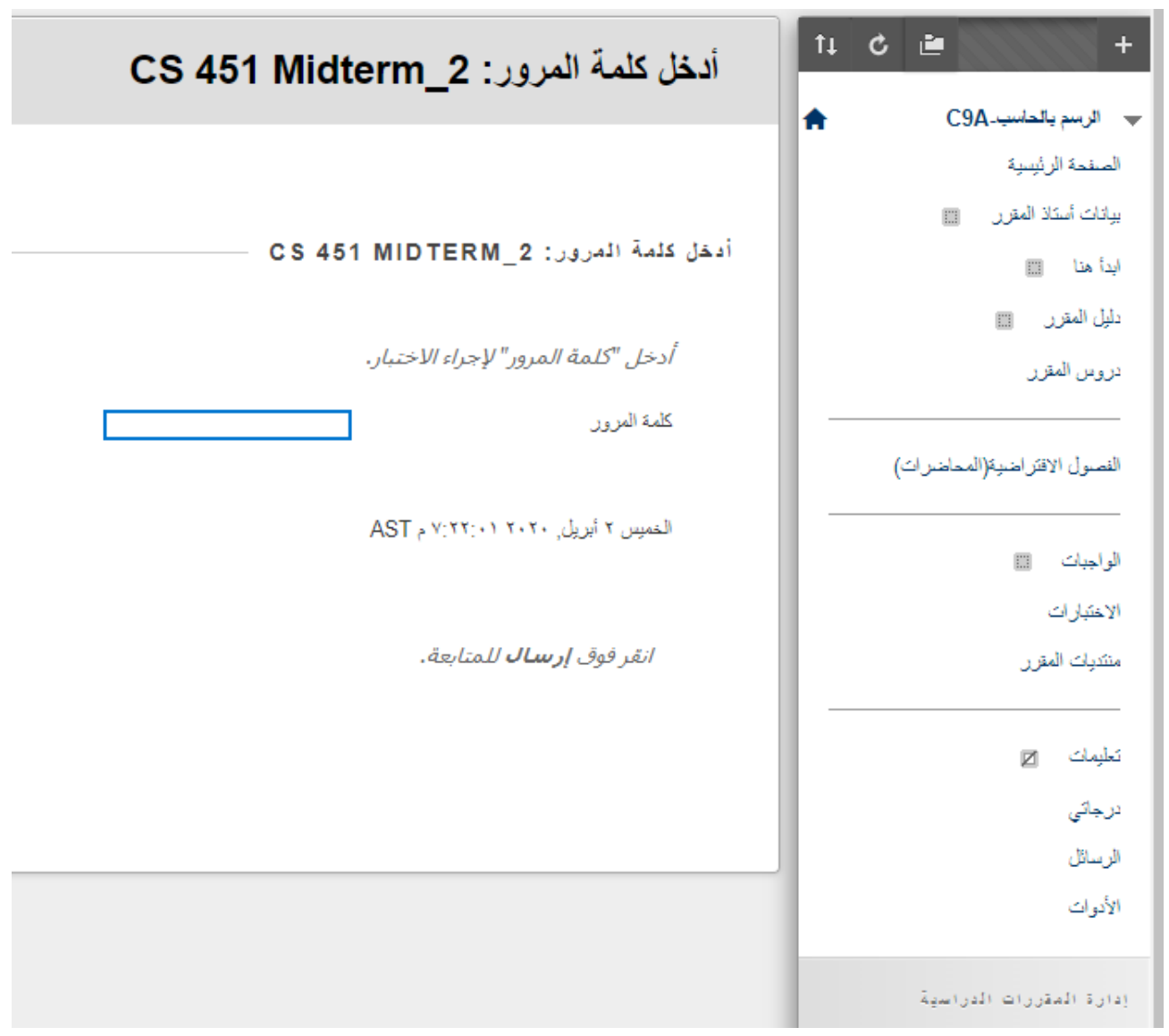

Figure 3. Entering the online exam password.

According to the university rules, entering the online exam password is done by the online exam proctor not by the exam taker. There are some drawbacks for this process. These drawbacks are summarized as follows:

1. Time consuming

2. Missed type

3. No fairness among exam takers in a room of 30 devices where one proctor needs to enter the password one by one.

4. A twin of the exam taker can conduct the online exam instead of the real exam taker due to the similarities in face.

In order to overcome such drawbacks, this paper proposes a new framework for ensuring real authentication for exam takers. This proposed framework uses the biometric authentication for ensuring the real authentication. The proposed system is presented in section 2. Section 3 presents the feature extraction method. Section 4 presents the classification phase. Section 5 presents the experimental results and the performance of the proposed system. Section 6 reviews the conclusion of this paper.

The remainder of this paper is organized as follows: Section 2 briefly presents an overview of optimization algorithms and opposition-based learning application. Section 3 explains the standard simulated Kalman filter algorithm, the concept of opposition-based learning and the proposed enhance version of SKF. Section 4 provides the experimental settings and discusses the experimental results. Section 5 concludes the paper.

\section{RELATED WORK}

In this paper, the proposed framework consists of the tradition recognition phases which are: image acquisition, the pre-processing, feature extraction, and classification. Figure 4 illustrates the proposed framework. 


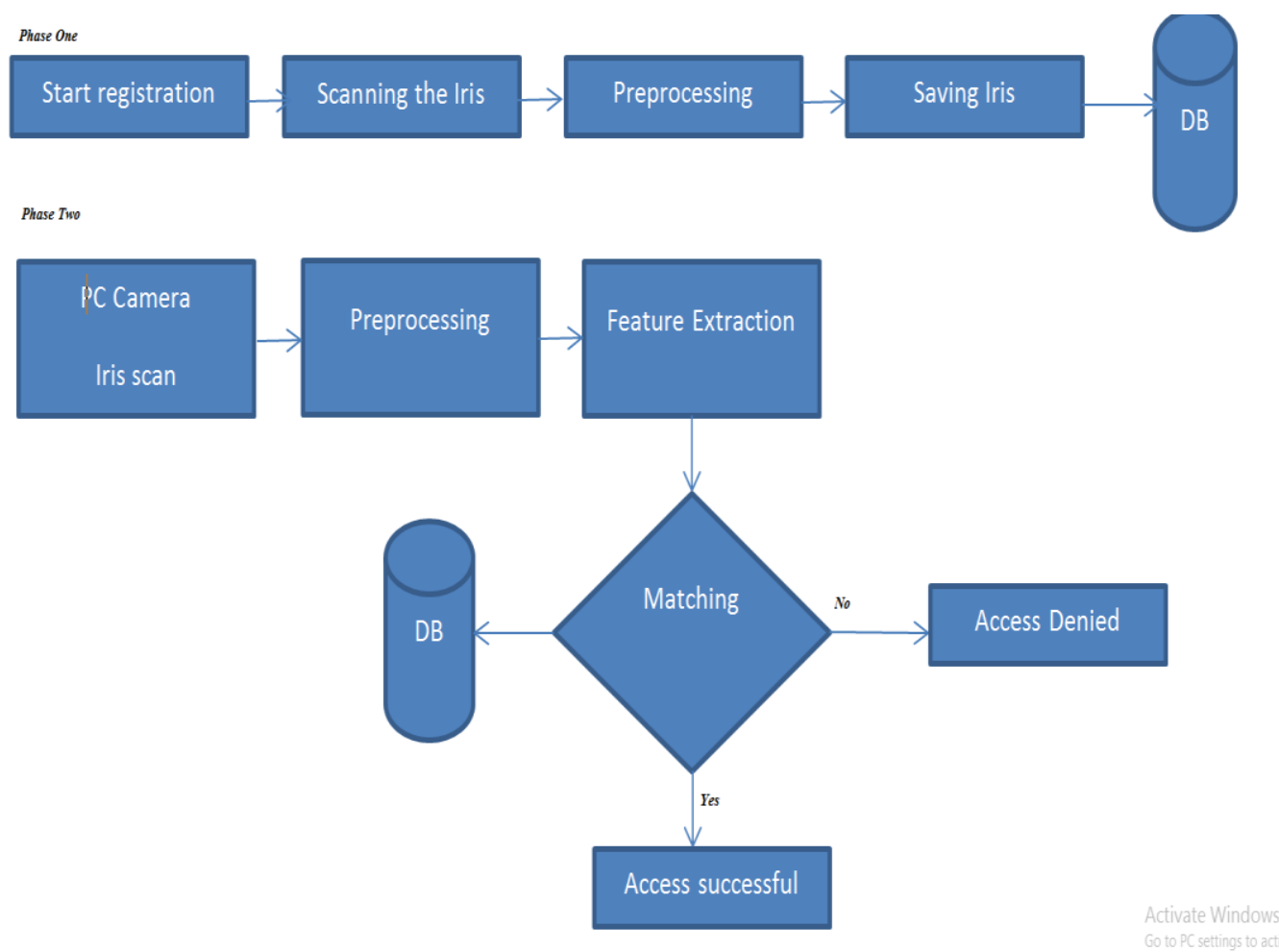

Figure 3. The proposed framework.

In general, both the user name and the associated password are used as an authentication in various systems. Basically, there are advantages and disadvantages for such systems. The only advantage for such systems, that they do not require extra hardware devices for capturing biometric features. The hardware can be camera, finger print scanning device, etc. In contrast, the disadvantages can be summarized as follows:

1. The security level depends on the mechanism of the user's ability in choosing and maintaining the password.

2. The system may reach a risk in the security level if the use forgets his/her password.

3. Moree security risk may occur in the system, if the intruder gets the user's password. This case occurs when all usernames and their associated passwords are saved.

According to the above disadvantages, this paper proposes a new authentication framework for ensuring the online exam takers. Basically, a new biometric factor is added to the existing online exam process at Taibah University. This addition enhances and improves the existing online exam process. The main goal for adding the new factor is making the Taibah University online exams process more robust. Mainly, the student is admitted to Taibah University and he/she is assigned a student number. Here, the student is asked to go the admission office for scanning his/her eye iris. Hence, similar pre-processing steps are applied to the student iris (Alkahteeb et al 2011). The student iris will be saved and stored in the Taibah University computer server with a private key which the student number (Alkhateeb Jawad 2107).

Another security risk would occur, if the intruder gets the password where all username and password are saved. By implementing the proposed framework, the process of conducting an online exam at Taibah University is summarized as follows:

1. The student exam taker login in into his/her blackboard account as illustrated in figure 1. It is the same of the existing system.

2. The camera of the computer device captures the iris of the student exam taker. Here, a comparison is done by the new iris captured image with the one stored in the Taibah University computer server. If the result of the comparison is true, the student exam taker will continue his/her exam towards step 3 where the screen opens for conducting the exam. Otherwise, the process of conducting the online exam will be terminated and the screen will be locked.

3. After a successful blackboard login in both ways: the user names with iris authentication, the student goes to his/her course in order to choose the required exam by clicking on it as illustrated in figure 2, and open the exam without password as required in the existing system. 


\section{FEATURE EXTRACTION METHOD}

The main goal of the feature extraction phase is to represent the digital image effectively by removing any redundant data. In this paper, the coefficients of the Discrete Cosine Transform (DCT) have been extracted as the distinctive features for the iris of the student exam taker. By applying the two dimensional DCT (2D_DCT) to the whole iris image, the features are extracted by using the coefficient of the 2D_DCT. Figure 5 shows general views of the iris and its parts (Sarhan Ahmad 2009).
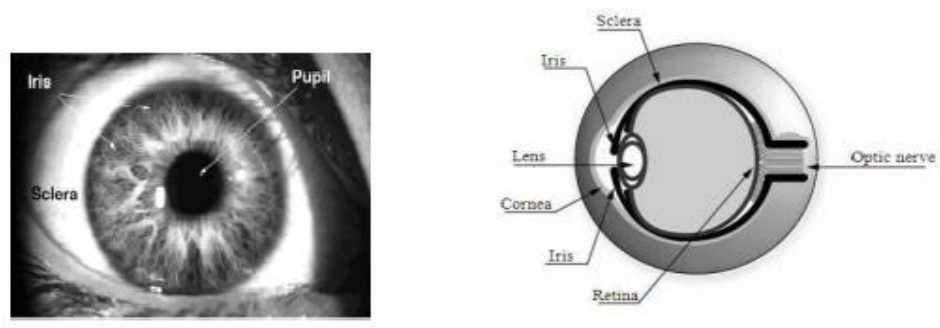

Figure 3. General views of the iris (Sarhan Ahmad 2009).

The2D-DCT is generated using Equation 1 (Alkhateeb et al 2011).

$$
f(u, v)=\alpha(u) \alpha(v) \sum_{i=0}^{I-1} \sum_{j=0}^{J-1} f(i, j) \cos \left[\frac{(2 i+1)}{2 I}\right] \cos \left[\frac{(2 j+1)}{2 J}\right]
$$

The number of extracted features has been chosen according to the energy reservation concept of the 2D-DCT. The energy is generated using Equation 2 (Alkhateeb et al 2011).

$$
E=\sum_{u=0}^{U-1} \sum_{v=0}^{V-1} f^{2}(u, v)
$$

By applying the 2D_DCT to the whole iris image is resulting into a two dimensional matrix known as DCT matrix as illustrated in figure $\overline{5}$.
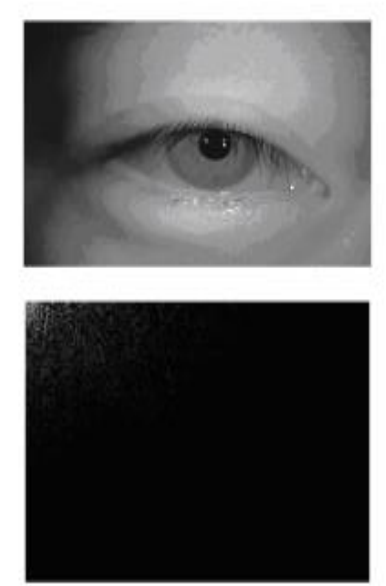

Figure 5. Iris image with its DCT.

The DCT matrix needs to be converted into one dimensional vector by using the zigzag order. Basically there are two different ways for converting the DCT matrix into one dimensional vector as illustrated in figure 6. Figure 6(b) is used in this paper. Most of the energy is found in the DC value of the DCT matrix. The number of the extracted DCT coefficients has been chosen to be 15 [7]. 


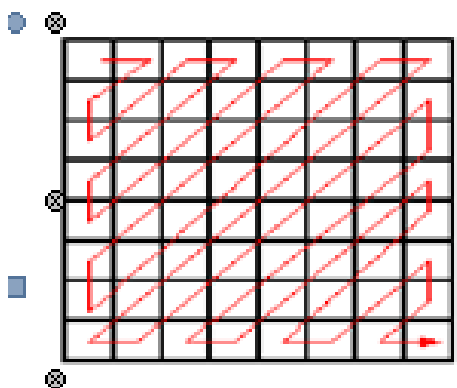

(a)
(

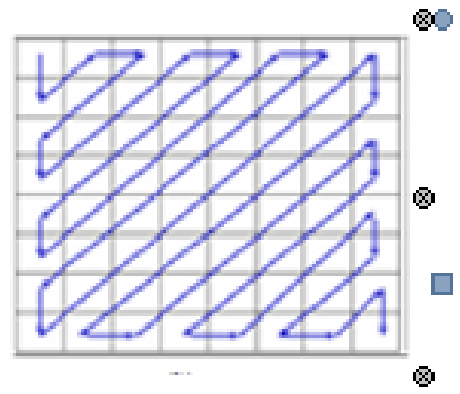

(b)

Figure 6. Two zigzag orders in sorting 2D DCT coefficients into one vector (Alkhateeb et al 2011). .

\section{CLASSIFICATION}

In general, the classification process is classified into two categories: Binary and Multi classification. In binary classification, there are two classes only. In contrast, the multi class classification has $n$ classes where $n>=3$. In classification phase, the extracted features are mapped into a classifier. In classification phase, there are two main learning approaches: the supervised learning and the unsupervised learning approaches. The supervised learning is the training system towards a given target. However, the unsupervised learning approach is the training without having a target by finding a relationship among various input features (Alkhateeb et al 2011).

In this paper, the traditional Artificial Neural Network (ANN) is employed to classify the extracted DCT features. Mainly, the ANN consists of a large number of input features which are highly connected together in order to solve a specific problem or task.

The ANN s used in this paper has the following specifications:

1. The feed forward network multi-layer (MLP) architecture.

2. Back propagation (BP) is used as a learning algorithm. It is a supervised learning approach.

3. Log sigmoid functions were used as the transfer function for the output layer.

\section{EXPERIMENTAL RESULTS}

As mentioned earlier, the process of implementing the proposed framework the process of conduction the online exam has two main steps: step 1 and step2. In step 2, a matching mechanism is done after scanning the iris of the online exam taker. If the matching is true, the screen is opened for the online exam taker to conduct the exam. Otherwise, the screen will be locked and the system will be terminated.

The DCT features were mapped into the traditional ANN classifier and the results were attractive. Ten different experiments were done using one computer device with a small database, and the recognition rate is taken out using the average of the ten experiments. The recognition rate was $82.5 \%$.. Figure 7 summarizes the recognition rate for the various ten experiments and their average. In addition, Table 1 summarizes the result the ten conducted experiments.

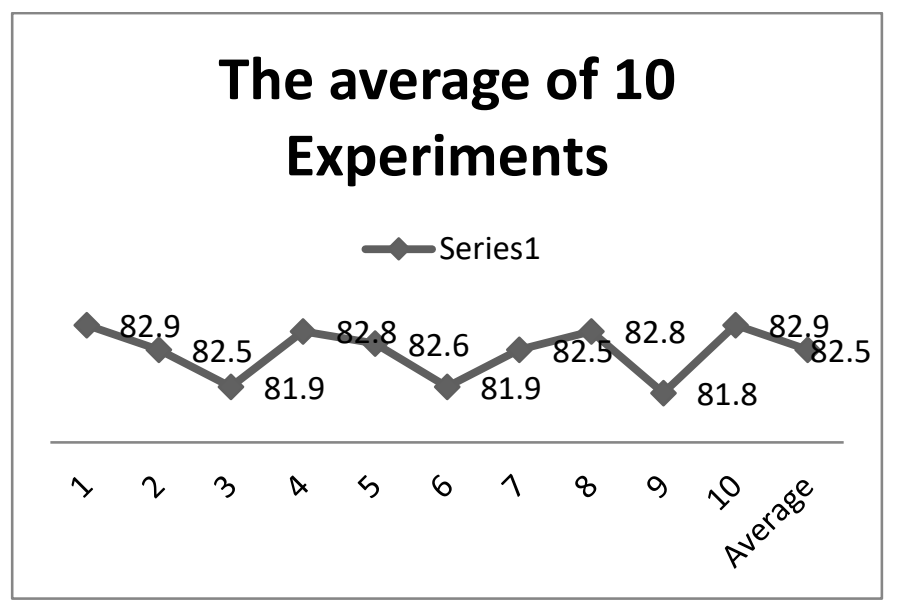

Figure 7. The result for each experiment and their average. 
Table 1. The result of various ten different experiments

\begin{tabular}{|c|c|}
\hline Experiment No. & Recognition Rate \% \\
\hline 1 & 82.9 \\
\hline 2 & 82.5 \\
\hline 3 & 81.9 \\
\hline 4 & 82.8 \\
\hline 5 & 82.6 \\
\hline 6 & 81.9 \\
\hline 7 & 82.5 \\
\hline 8 & 82.8 \\
\hline 9 & 81.8 \\
\hline 10 & 82.9 \\
\hline Average & 82.5 \\
\hline
\end{tabular}

\section{CONCLUSION}

In this paper, a new framework for ensuring the online exams authentication is proposed. The system uses the 2D_DCT and the ANNs for training and testing. The results of the proposed framework and its performance based on ANN classifier show an attractive result of $85.5 \%$ as recognition rate.

\section{REFERENCES}

Alkhateeb, Jawad (2018). A New Model for Enhancing Computer Based Exam Authentication in KSA. Oriental Journal of Computer Science and Technology 11(1): 12-17.

Alkhateeb, Jawad (2017). A Database For Identifying the Missed Pilgrims in Hajj and Umrah. International Journal of Computer and Information Technology (IJCIT) 6 (6): 346-351.

Alkhateeb, Jawad, et al. (2011). Offline handwritten Arabic cursive text recognition using Hidden Markov Models and re-ranking. Patteren Recognition Letters 32(8): 1081-1088.

Alkhateeb, Jawad , et al. (2011). Performance of hidden Markov model and dynamic Bayesian network classifiers on handwritten Arabic word recognition. Knowledge-Based Systems 24(5): 680-688.

Kanimozhi, k. (2015). Biometric Authentication for Online Examination. International Journal of Computer Science and Engineering 3(9): 162-164.

Ramu, T and Arivoli. (2013). A Framework of Secure Biometric Based Online Exam Authentication: An Alternative to traditional Exam. International Journal of Science \& Engineering Research 4(11): 52-60.

Sarhan Ahmad (2009). Iris Recognition Using Discete Cosine Transform and Artificial Neyral Networks. Journal of Computer Science 5 (5): 369-373.

Traore Isaa, et al. (2017). Ensuring Online Exam Integrity Through Continous Biometric Authentication. Springer International Publishing AG Chapter 6: 73-81.

\section{ACKNOWLEDGEMENT}

Sincere thanks and appreciation are expressed to the TAIBAH University, College of Computer Science and Engineering (CCSE), Kingdom of Saudi Arabia for supporting this research. 